\title{
Self-Assembling Nanofibers Inhibit Glial Scar Formation and Promote Axon Elongation after Spinal Cord Injury
}

\author{
Vicki M. Tysseling-Mattiace, ${ }^{1 \star}$ Vibhu Sahni, ${ }^{1 \star}$ Krista L. Niece, ${ }^{3}$ Derin Birch, ${ }^{1}$ Catherine Czeisler, ${ }^{1}$ Michael G. Fehlings, ${ }^{4}$ \\ Samuel I. Stupp, ${ }^{2,3}$ and John A. Kessler ${ }^{1}$ \\ ${ }^{1}$ Department of Neurology and ${ }^{2}$ Department of Medicine and Institute for BioNanotechnology in Medicine, Northwestern University, Chicago, Illinois \\ 60611, ${ }^{3}$ Departments of Materials Science and Engineering and Chemistry, Northwestern University, Evanston, Illinois 60208, and ${ }^{4}$ Department of Surgery, \\ University of Toronto, Toronto, Ontario, Canada M5S 1A8
}

Peptide amphiphile (PA) molecules that self-assemble in vivo into supramolecular nanofibers were used as a therapy in a mouse model of spinal cord injury (SCI). Because self-assembly of these molecules is triggered by the ionic strength of the in vivo environment, nanoscale structures can be created within the extracellular spaces of the spinal cord by simply injecting a liquid. The molecules are designed to form cylindrical nanofibers that display to cells in the spinal cord the laminin epitope IKVAV at nearly van der Waals density. IKVAV PA nanofibers are known to inhibit glial differentiation of cultured neural stem cells and to promote neurite outgrowth from cultured neurons. In this work, in vivo treatment with the PA after SCI reduced astrogliosis, reduced cell death, and increased the number of oligodendroglia at the site of injury. Furthermore, the nanofibers promoted regeneration of both descending motor fibers and ascending sensory fibers through the lesion site. Treatment with the PA also resulted in significant behavioral improvement. These observations demonstrate that it is possible to inhibit glial scar formation and to facilitate regeneration after SCI using bioactive three-dimensional nanostructures displaying high densities of neuroactive epitopes on their surfaces.

Key words: spinal cord injury; nanotechnology; gliosis; regeneration; extracellular matrix; functional recovery

\section{Introduction}

Spinal cord injury (SCI) often leads to permanent paralysis and loss of sensation below the site of injury because of the inability of damaged axons to regenerate in the adult CNS. Various approaches have been used to treat SCI with notable but, unfortunately, limited success (Thuret et al., 2006). We report here on the use of a molecularly designed bioactive matrix without exogenous proteins or cells as a different therapeutic approach for SCI. The matrix is composed of peptide amphiphile (PA) molecules that self-assemble from aqueous solution into cylindrical nanofibers that display bioactive epitopes on their surfaces (Hartgerink et al., 2001, 2002; Silva et al., 2004) (Fig. 1; supplemental Figs. 1-3, available at www.jneurosci.org as supplemental material). The cylinders have a well defined diameter in the range of $6-8 \mathrm{~nm}$ and consist of $\beta$-sheet assemblies that tend to be parallel to the nanofibers (Jiang et al., 2007). Because each molecule con-

Received Nov. 1, 2007; revised March 5, 2008; accepted March 7, 2008.

This project was supported by National Institutes of Health Grants R01 EB003806-01 (S.I.S., J.A.K.), R01 NS20013-21 (J.A.K.), and P50 NS54287 (J.A.K.) and by a scholarship from the Foundation for Physical Therapy (V.M.T.). We thank Bridgid Nolan, Tammy McGuire, and Min Hu for assistance with animal care, Hongzhou Jiang for help with the scanning electron microscopy, and Jie Huang for help with statistics. We thank 0 swald Steward and his laboratory for advice and training on the tract-tracing methodology.

S.S. has founded a company, Nanotope, to eventually develop commercial applications of the self-assembling materials. S.I.S. and J.A.K. participate in this venture.

*V.M.T.-M. and V.S. contributed equally to this work.

Correspondence should be addressed to Vicki M. Tysseling-Mattiace, 303 E. Chicago Avenue, Chicago, IL 60611. E-mail:v-mattiace@northwestern.edu.

DOI:10.1523/JNEUROSCI.0143-08.2008

Copyright $\odot 2008$ Society for Neuroscience $\quad 0270-6474 / 08 / 283814-10 \$ 15.00 / 0$ tains an epitope sequence at its hydrophilic terminus, the nanostructures assembled in an aqueous medium are able to display bioactive sequences perpendicular to their long axis at nearly van der Waals density. This intensifies the epitope density compared with laminin by a factor of $10^{3}$ (Silva et al., 2004).

We reported previously on a negatively charged PA incorporating the neuroactive pentapeptide epitope from laminin, isoleucine-lysine-valine-alanine-valine (IKVAV) (Silva et al., 2004). Addition of physiological fluids to dilute aqueous solutions of this PA leads to spontaneous formation of nanofibers in vitro and in vivo (Silva et al., 2004). In our previous studies, the nanofibers containing the IKVAV epitope were found to promote outgrowth of processes from cultured neurons and to suppress astrocytic differentiation of cultured neural progenitor cells (Silva et al., 2004). The suppression of astrocytic differentiation was not observed when using a simple IKVAV peptide, the protein laminin, or a control PA that did not contain the neuroactive epitope and that instead had the nonbioactive sequence EQS at one terminus (EQS PA) (Silva et al., 2004). Furthermore, the PA promoted copious neurite outgrowth that far exceeded what was observed with either laminin or IKVAV peptide. Thus, the biological effects of the IKVAV PA differ strikingly from those of either laminin or IKVAV peptide. These observations suggested that injection of the bioactive amphiphile after SCI might reduce astrogliosis and possibly promote axon outgrowth.

\section{Materials and Methods}

Mouse spinal cord injuries, amphiphile injections and animal care. All animal procedures were undertaken in accordance with the Public Health 

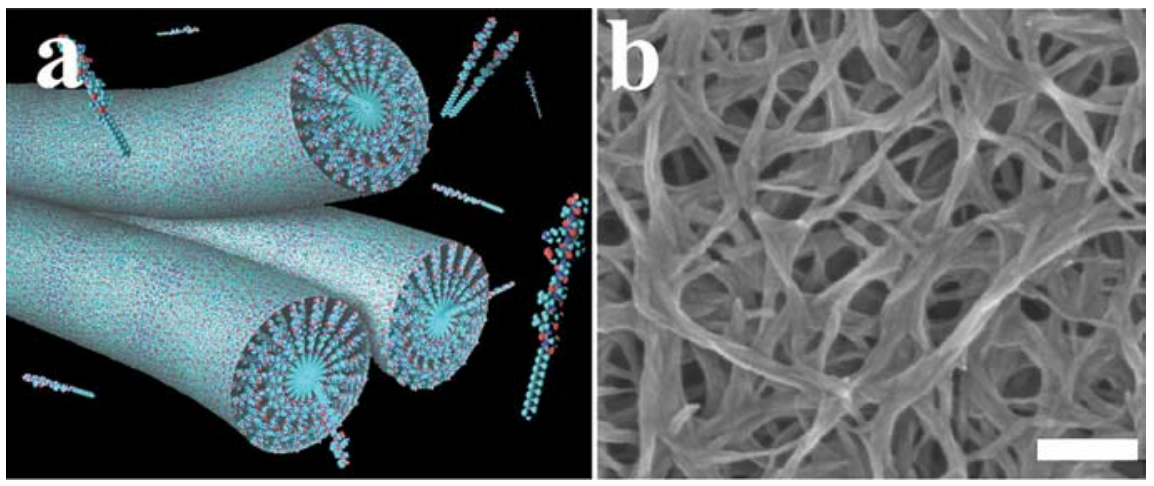

Figure 1. Structure of IKVAV PA. $\boldsymbol{a}$, Schematic representation showing individual PA molecules assembled into a bundle of nanofibers interwoven to produce the IKVAV PA. $\boldsymbol{b}$, Scanning electron micrograph image shows the network of nanofibers in vitro. Scale bar, $200 \mathrm{~nm}$.

Service Policy on Humane Care and Use of Laboratory Animals. The Institutional Animal Care and Use Committee approved all procedures. Female 129 SvJ mice (10 weeks of age; The Jackson Laboratory, Bar Harbor, ME) were anesthetized using Avertin intraperitoneally. After laminectomy at the T10 vertebral segment, the spinal cord was compressed dorsoventrally by the extradural application of a 24 g modified aneurysm clip for 1 min (FEJOTA mouse clip). After SCI, the skin was sutured using AUTOCLIP (9 mm; BD Biosciences, San Jose, CA). Postoperatively, animals were kept under a heat lamp to maintain body temperature. A $1.0 \mathrm{cc}$ injection of saline was given subcutaneously, which was repeated daily for the first week after the injury. Bladders were manually emptied twice daily throughout the duration of the study. In the event of discomfort, buprenex ( $2 \mathrm{mg} / \mathrm{kg}$, s.c., twice daily) was administered. Gentamycin was administered once daily in the event of hematuria $(20 \mathrm{mg} /$ $\mathrm{kg}$ ) subcutaneously for $5 \mathrm{~d}$. Mice that exhibited any hindlimb movement $24 \mathrm{~h}$ after the injury were excluded from the study.

PA ( $1 \%$ aqueous solution) or vehicle was injected $24 \mathrm{~h}$ after SCI using borosilicate glass capillary micropipettes (Sutter Instruments, Novato, CA) (outer diameter, $100 \mu \mathrm{m}$ ) coated with Sigmacote (Sigma, St. Louis, MO) to reduce surface tension. The capillaries were loaded onto a Hamilton syringe using a female luer adaptor (World Precision Instruments, Sarasota, FL) controlled by a Micro4 microsyringe pump controller (World Precision Instruments). The amphiphile was diluted 1:1 with a $580 \mu \mathrm{M}$ solution of glucose just before injection and loaded into the capillary. Under Avertin anesthesia, the autoclips were removed and the injury site was exposed. The micropipette was inserted to a depth of 750 $\mu \mathrm{m}$ measured from the dorsal surface of the cord, and $2.5 \mu \mathrm{l}$ of the diluted amphiphile solution or vehicle was injected at $1 \mu \mathrm{l} / \mathrm{min}$. The micropipette was withdrawn at intervals of $250 \mu \mathrm{m}$ to leave a trail (ventral to dorsal) of the IKVAV PA in the cord. At the end of injection, the capillary was left in the cord for an additional $5 \mathrm{~min}$, after which the pipette was withdrawn and the wound closed. For all experiments, the experimenters were kept blinded to the identity of the animals.

Animal perfusions and tissue acquisition. Animals were killed using an overdose of halothane anesthesia and transcardially perfused with $4 \%$ paraformaldehyde in PBS. The spinal cords were dissected and fixed overnight in 30\% sucrose in 4\% PFA. The spinal cords were then frozen in Tissue-Tek embedding compound and sectioned on a Leica (Deerfield, IL) CM3050S cryostat. Longitudinal sections (20 $\mu \mathrm{m}$ thick) were taken.

GFAP quantitation. Sections were rinsed with PBS twice and then incubated with anti-glial fibrillary acidic protein (GFAP; 1:250; mouse monoclonal IgG1; Sigma) for $1 \mathrm{~h}$ at room temperature. After this, sections were rinsed thrice with PBS and incubated with Alexa Fluorconjugated anti-mouse IgG1 secondary antibodies (1:500; Invitrogen, Carlsbad, CA) for $1 \mathrm{~h}$ at room temperature. Sections were finally rinsed thrice with PBS and then incubated with Hoechst nuclear stain for 10 min at room temperature. After a final rinse with PBS, they were mounted using Prolong Gold anti-fade reagent (Invitrogen) and imaged using a Zeiss (Thornwood, NY) UVLSM-Meta confocal microscope. Af- ter immunostaining, we measured the fluorescence intensity of GFAP immunoreactivity to estimate the fold increase in GFAP levels around the lesion over baseline levels in uninjured parts of the cord. We also compared the GFAP fluorescence intensity to levels in spinal cord of normal uninjured animals. For each animal, sections at equivalent mediolateral depth were used for analysis. Because we knew the right-left thickness of each spinal cord, we could pick sections at the equivalent mediolateral depths in different animals very accurately. Furthermore, the mediolateral thickness of the cord was not observed to be different between IKVAV PAinjected and control animals. Confocal scans in the lesioned area were taken immediately adjacent to the area of peak infiltration (supplemental Fig. 4, available at www.jneurosci.org as supplemental material), where we observed the most intense GFAP immunoreactivity. Each scan was performed using identical laser power, gain, and offset values. These values were set such that the pixels in the images of the lesioned area did not saturate, and those in uninjured areas were not too dim (intensity sufficiently above zero). $Z$-stacks of the scans were reconstructed using LSM image browser (Zeiss). Fluorescence quantitation was performed by converting the entire $Z$-stack into a monochrome (.tif) image and subsequently measuring the intensity of each pixel. Each pixel has a grayscale that ranges from 0 to 255 . The total pixel intensity of each stack was integrated using the MetaMorph 2.6 software. Intensity values at the lesioned area for each individual section were normalized to the baseline values derived from scans taken over uninjured parts of the same section, which we have defined as $1 \mathrm{~mm}$ away from the edge of the area of increased GFAP immunoreactivity. We also normalized the intensity of gliosis at the lesion site to the values obtained from an uninjured cord (supplemental Fig. 6, available at www.jneurosci.org as supplemental material). For each section, four sites (two rostral and two caudal to the lesion epicenter) in the lesioned area and three in the uninjured area (spanning both gray and white matter) were scanned. At least four sections were analyzed for each animal in such a manner. The total intensity values were then averaged for each group. The final fluorescence values were therefore expressed as fold increases over the baseline (uninjured area) values for individual sections, which were then grouped for each animal for comparison between IKVAV PA and vehicle-injected groups.

Apoptotic cell death and oligodendrocyte density quantification. Sections were boiled in $10 \mathrm{~mm}$ sodium citrate for $20 \mathrm{~min}$ and then cooled to room temperature. They were then incubated in blocking solution [PBS containing $10 \%$ BSA, $1 \%$ normal goat serum (NGS), and $0.25 \%$ Triton $\mathrm{X}-100]$ for $1 \mathrm{~h}$ at room temperature. After this, the sections were incubated in primary antibody solution (PBS with $1 \%$ NGS and $0.25 \%$ Triton $\mathrm{X}-100)$ overnight at $4^{\circ} \mathrm{C}$. After three $1 \times$ PBS washes on the next day, the sections were incubated with the appropriate secondary antibody (1:500) for $1 \mathrm{~h}$ at room temperature and then rinsed with PBS and stained with Hoechst nuclear stain as already described above. Antibodies used were anti-APC/mAb CC1 (1:200; EMD Biosciences, San Diego, CA) and anticleaved caspase-3 (1:200; Cell Signaling Technology, Danvers, MA).

Similar to the quantification of GFAP levels, sagittal sections in all animals were taken at the equivalent mediolateral depths. The lesion was defined as the area marked by dense infiltration, and apoptotic cells were counted both in the epicenter and $400 \mu \mathrm{m}$ rostral and caudal to it. CC1positive cells were counted $400 \mu \mathrm{m}$ rostral and caudal to the lesion. The area analyzed spanned the entire dorsoventral thickness of the cord.

Tract tracing and analysis. At $1 \mathrm{~d}$ or 9 weeks after injury, mice were anesthetized with Avertin and were injected with mini-ruby-conjugated BDA (Invitrogen) using a $10 \mu \mathrm{l}$ Hamilton microsyringe fitted with a pulled-glass micropipette. For dorsal column labeling, $2 \mu$ l were injected just distal to the L5 dorsal root ganglion. The corticospinal tract was labeled with three injections $(0.5 \mu \mathrm{l}$ each) made at $1.0 \mathrm{~mm}$ lateral to the midline at $0.5 \mathrm{~mm}$ anterior, $0.5 \mathrm{~mm}$ posterior, and $1.0 \mathrm{~mm}$ posterior to bregma, and at a depth of $0.5 \mathrm{~mm}$ from the cortical surface. Animals were 

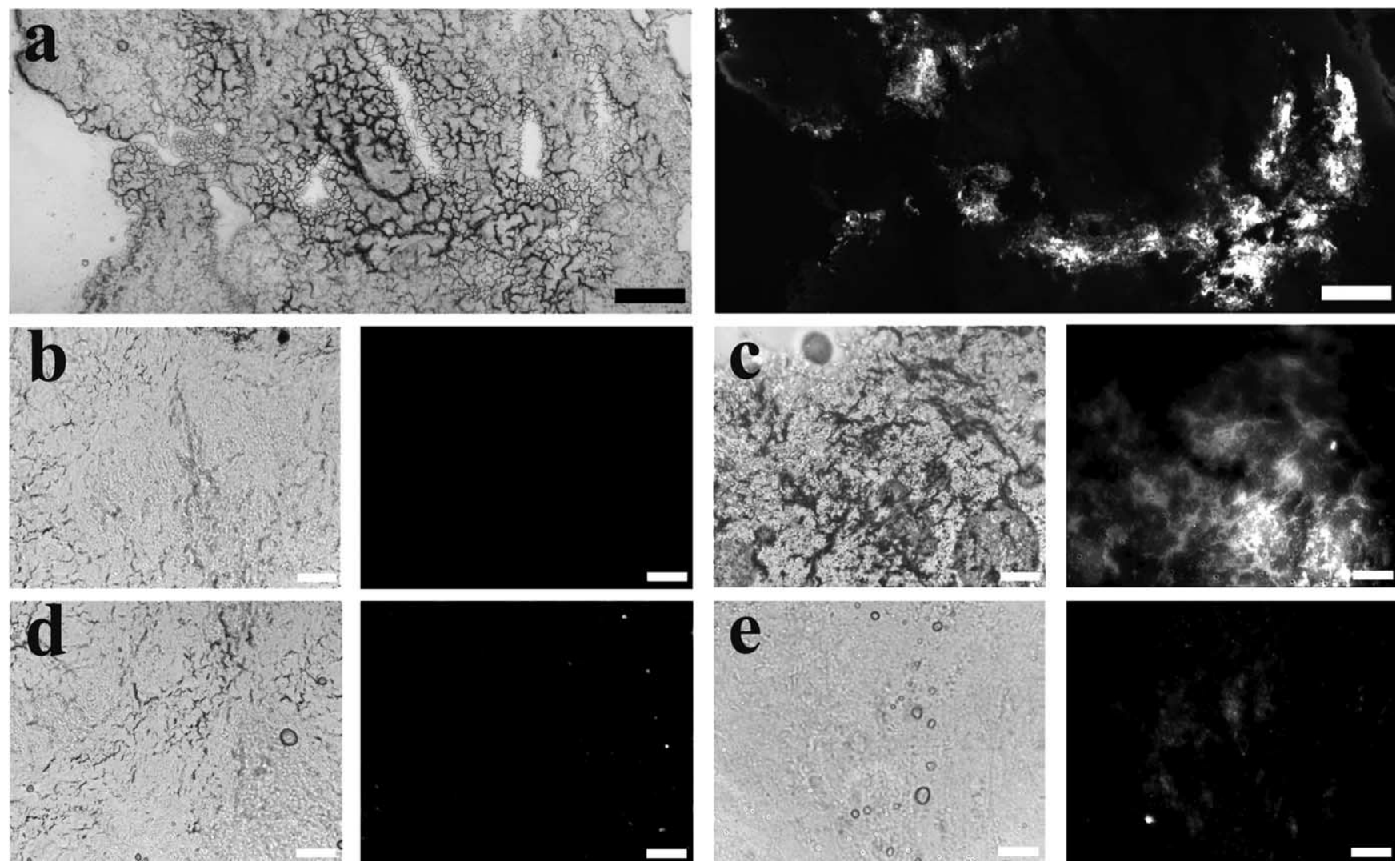

Figure 2. IKVAV PA solution self-assembles in vivo. $\boldsymbol{a}$, Phase (left) and fluorescent (right) images showing the fluorescent IKVAV PA in the injured spinal cord $24 \mathrm{~h}$ after injection (dorsal, left; rostral, top). $\boldsymbol{b}-\boldsymbol{e}$, Longitudinal sections of spinal cord showing the fluorescent IKVAV PA is present at 2 weeks after injection only in IKVAV PA-injected animals (c) versus control (uninjected; $\boldsymbol{b}$ ), but by 4 weeks, the IKVAV PA has mostly biodegraded, as seen in the IKVAV PA-injected animals (e) compared with uninjected animals (d). Scale bars: $\boldsymbol{a}, 100 \mu \mathrm{m}, \boldsymbol{b}-\boldsymbol{e}, 50 \mu \mathrm{m}$.

killed $14 \mathrm{~d}$ after BDA injection and perfused. Floating $20 \mu \mathrm{m}$ sagittal serial sections were each collected and washed three times in $1 \times$ PBS and $0.1 \%$ Triton $\mathrm{X}-100$, incubated overnight at $4^{\circ} \mathrm{C}$ with avidin and biotinylated horseradish peroxidase (Vectastain ABC Kit Elite; Vector Laboratories, Burlingame, $\mathrm{CA}$ ), washed again three times in $1 \times \mathrm{PBS}$, and then reacted with $\mathrm{DAB}$ in $50 \mathrm{~mm}$ Tris buffer, $\mathrm{pH}$ 7.6, 0.024\% hydrogen peroxide, and $0.5 \%$ nickel chloride. Every section was collected serially so that individual axons could be traced from section to section. Sections were then transferred to PBS and mounted in serial order on microscope slides using a weak mounting media containing $0.1 \%$ gelatin and $10 \%$ ethanol in PBS. Tracts were traced using Neurolucida imaging software (MicroBrightField, Williston, VT) for each axon that was labeled within a $500 \mu \mathrm{m}$ distance rostral to the lesion for corticospinal tract analysis and caudal to the lesion for dorsal column analysis.

\section{Results}

A unique feature of the PA is its ability to self-assemble into nanofibers when it is injected into tissue and contacts cations (Silva et al., 2004). To test the in vivo properties of the IKVAV PA as well as the therapeutic effects on recovery, we used a clip compression model of SCI that produces a consistent injury in mice where an initial impact is followed by persistent compression analogous to most cases of human SCI (Joshi and Fehlings, 2002a,b). Parameters were chosen to produce a particularly severe injury. Because a PBS buffer would trigger self-assembly and make the PA gel before injection, an isotonic glucose solution was used for injections of PA. We first tested the stability of the biodegradable IKVAV PA nanofibers. At $24 \mathrm{~h}$ after SCI, the lesion site was injected with a fluorescent derivative of the IKVAV PA (PA2 in supplemental Figs. 1-3, available at www.jneurosci.org as supplemental material). Longitudinal sections of spinal cord show that the fluorescent IKVAV PA is present at 2 weeks, but by 4 weeks, the IKVAV PA has mostly biodegraded. Therefore, the stability of the IKVAV PA was found to be on the order of weeks (Fig. 2).

\section{Functional recovery after SCI}

We used the Basso, Beattie and Bresnahan (BBB) locomotor scale modified for the mouse (Basso et al., 1996; Joshi and Fehlings, 2002a) to assess behavioral recovery from experimental SCI. For this and all subsequent experiments, the experimenters were kept blinded to the identity of the animals. At $24 \mathrm{~h}$ after SCI, the lesion site was injected with IKVAV PA, the nonbioactive EQS PA, or glucose, and some animals received sham injection. At 9 weeks after SCI, the EQS PA, sham injection, and glucose groups did not differ from one another, and all scored significantly less than the IKVAV PA group (Fig. 3a). Notably, injection of IKVAV peptide alone did not promote functional recovery (Fig. $3 b$ ), indicating that both the nanostructure of the PA and the IKVAV sequence are necessary for the beneficial effects on behavior. The nearly identical scores for the three control groups are also identical to those of animals in previous studies that received a complete transection of the spinal cord (Joshi and Fehlings, 2002a). A glucose control group was therefore used for comparison with IKVAV PA in all of the subsequent experiments in which animals were analyzed anatomically. In the behavioral analysis of the animals used for the anatomic studies, there were no distinguishable differences between the control and IKVAV PA groups until 5 weeks (Fig. 3c). However, at 5 weeks and thereafter, the IKVAV PA-injected group displayed significant behavioral improvement 


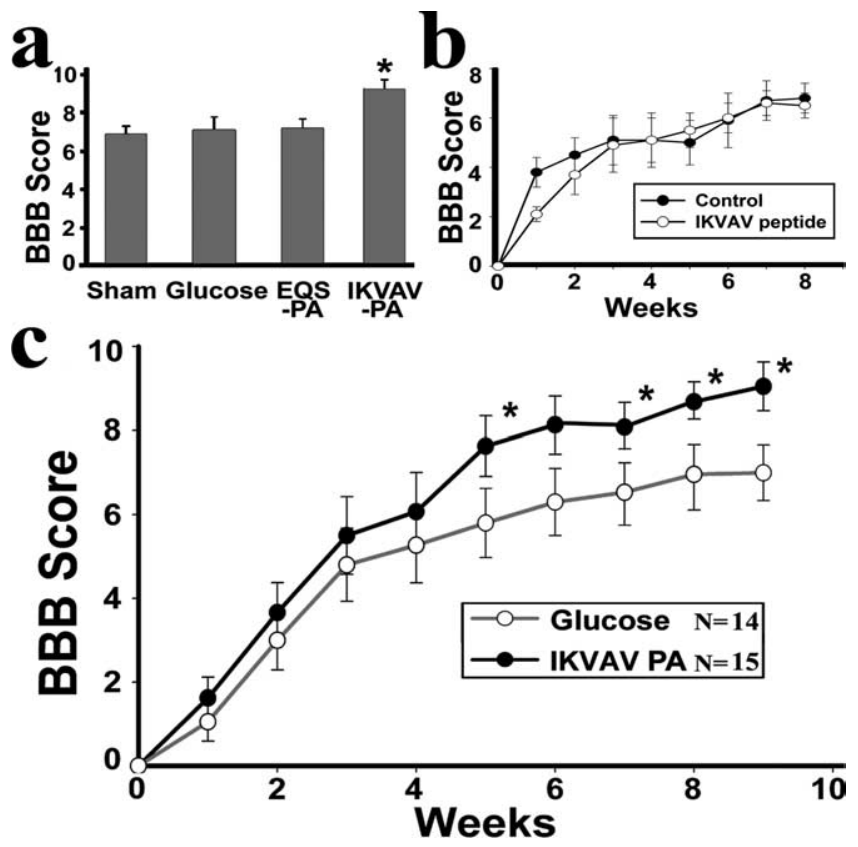

Figure 3. IKVAV PA promotes functional recovery as analyzed by the BBB scale. $\boldsymbol{a}$, Graph shows mean mouse $\mathrm{BBB}$ locomotor scores at 9 weeks after $\mathrm{SCl}$ for animals receiving injections of glucose, EQS PA, IKVAV PA, or sham injection. The IKVAV PA group differed from all others at $p<0.045 . n=7$. Because there was no difference among these controls, glucose was used in the subsequent experiment depicted in $\boldsymbol{c} . \boldsymbol{b}$, IKVAV peptide was injected at the same manner as the IKVAV PA. There were no significant differences in the BBB scores of animals injected with IKVAV peptide compared with sham controls. c, The graph shows mean mouse BBB locomotor scores between IKVAV PA and glucose injections after SCI. The IKVAV $(n=15)$ and glucose $(n=$ 14) groups differ from each other at $p<0.04$ by ANOVA with repeated measures. *Tukey's HSD post hoc $t$ tests showed that scores differed at $p<0.045$ at every time point 5 weeks after SCl and thereafter.

compared with the glucose control. At 9 weeks, the mean BBB score for the control group was $7.03 \pm 0.8$, whereas the mean score for the IKVAV PA-injected group was $9.2 \pm 0.5(p<0.04)$. Importantly, this represents significant functional recovery, because a score of 7 implies no functional movement despite an extensive range of movement in all three joints in the hindlimb, whereas a score of 9 indicates dorsal stepping in which the animal steps on the dorsal side of its foot during locomotion, i.e., the hindlimb movement has a functional use.

\section{Effects of IKVAV PA on astrogliosis}

Because the IKVAV PA produced significant long-term functional improvement, it seemed likely that it had altered cellular events, such as glial scar formation and cell death, that prevent recovery from SCI. Astrogliosis after neural injury involves an early hypertrophic (increased cell size) as well as a hyperplastic (increased cell number) response (Fawcett and Asher, 1999; Faulkner et al., 2004). At 4 d after SCI, GFAP immunohistochemistry revealed no apparent difference between the treated and control animals (Fig. 4a,d). However, at 5 weeks and 11 weeks after SCI, there was an obvious reduction in astrogliosis in the IKVAV PA-injected group (Fig. 4b,c). To quantify these changes, we measured the intensity of GFAP immunofluorescence in the lesion site normalized to baseline levels in the same cord $1 \mathrm{~mm}$ from the lesion (Fig. $4 d$ ) and found a significant reduction in the treated group $(2.56 \pm 0.6$ for IKVAV PA; $3.31 \pm 0.9$ for controls; $p<0.02$ at 5 weeks; and $2.0 \pm 0.1$ for IKVAV PA; $2.7 \pm 0.2$ for controls; $p<0.04$ at 11 weeks). In contrast, there was no difference in GFAP in animals that received nonbioactive PA (EQS)
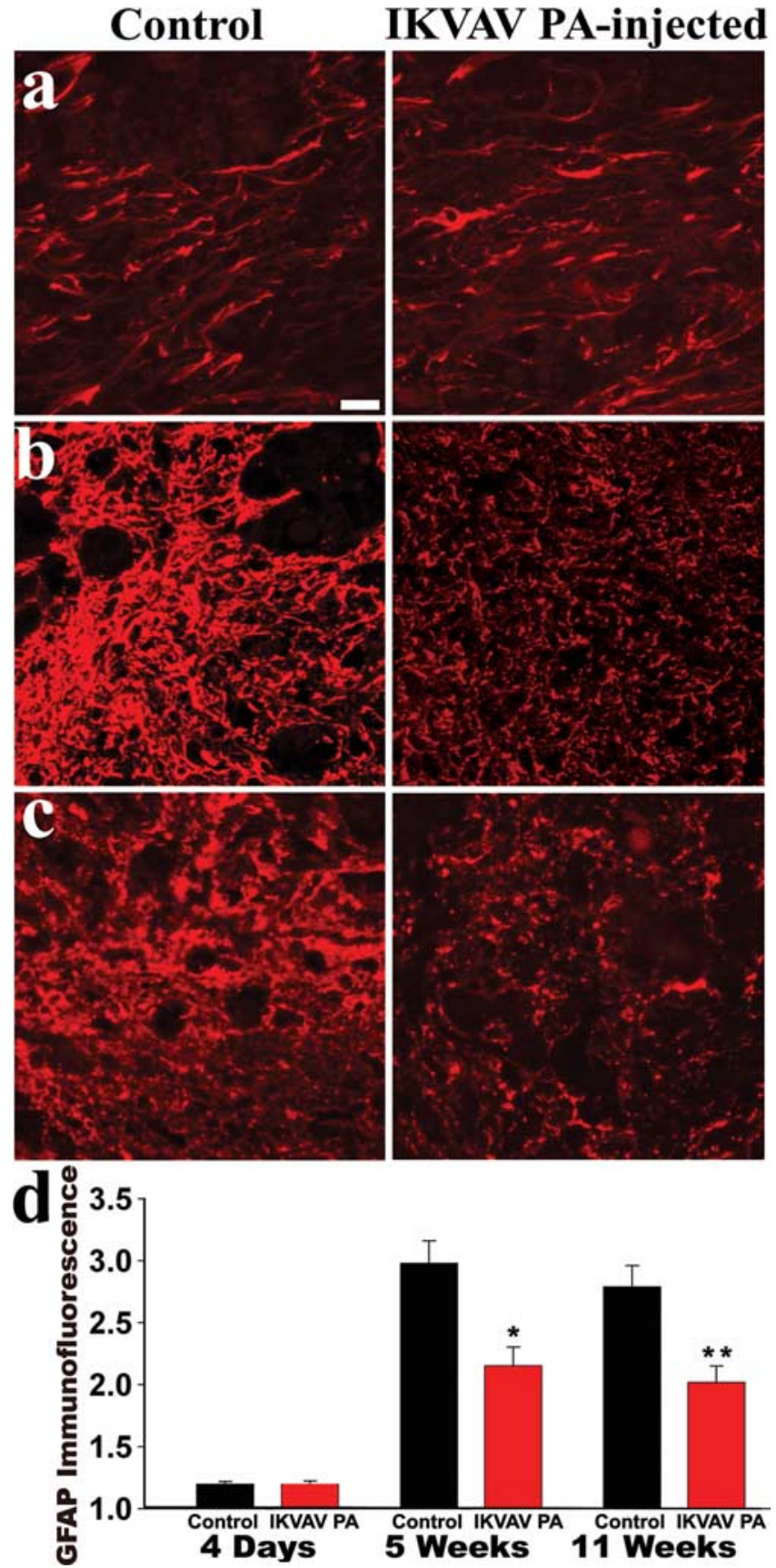

Figure 4. IKVAV PA attenuates astrogliosis in vivo after $\mathrm{SCl}$. $\boldsymbol{a}-\boldsymbol{c}$, Representative confocal $Z$-stacks of injured areas stained with GFAP in control and IKVAV PA-injected animals. The lesion is defined as the area marked by dense infiltration (0kada et al., 2006). The two groups do not differ at $4 \mathrm{~d}(\boldsymbol{a})$, but at 5 weeks $(\boldsymbol{b})$ and 11 weeks $(\boldsymbol{c})$, there is significantly less glial scarring in the IKVAV PA-injected animals. $\boldsymbol{d}$, GFAP immunofluorescence levels (expressed as fold increases over uninjured areas) in the IKVAVPA-injected animals are significantly reduced compared with control animals at 5 and 11 weeks ( ${ }^{*} p<0.02,{ }^{* *} p<0.04$ by $t$ test). Scale bar, $20 \mu \mathrm{m}$.

compared with glucose. Results were similar when the GFAP immunofluorescence was normalized to intensity values in normal, uninjured spinal cords (supplemental Fig. 6, available at www.jneurosci.org as supplemental material). Thus, injection of the IKVAV PA suppressed progression of astrogliosis at the lesion site but did not alter the initial reactive hypertrophy that may be essential for repairing the blood-brain barrier and restoring homeostasis (Faulkner et al., 2004; Okada et al., 2006). 


\section{Control}
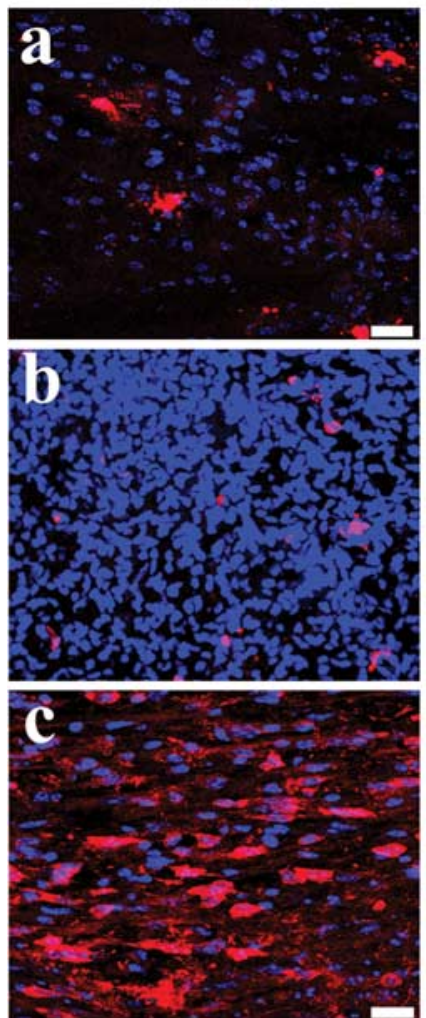

\section{IKVAV PA-injected}
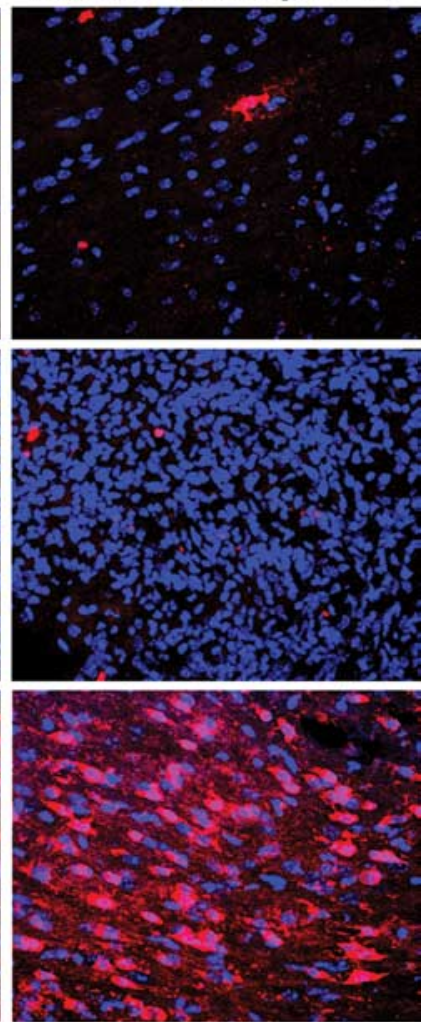

d

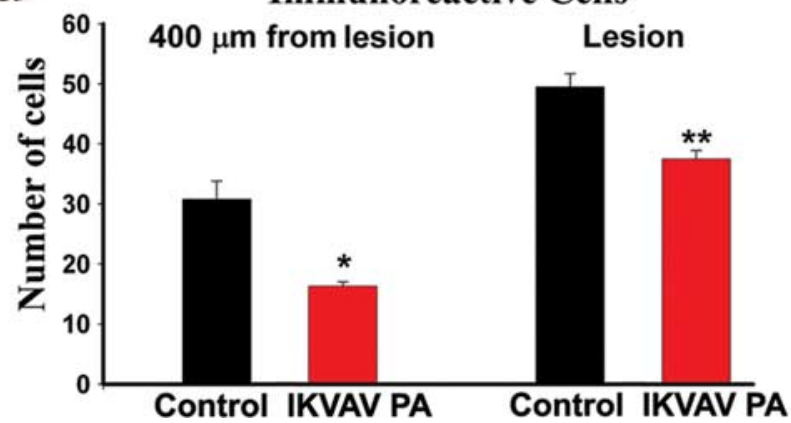

Figure 5. Apoptotic cell death is reduced in IKVAV PA-injected animals. $\boldsymbol{a}, \boldsymbol{b}$, Collapsed confocal Z-stacks of 20 - $\mu \mathrm{m}$-thick spinal cord sections (red, activated caspase-3; blue, Hoechst nuclear stain). The IKVAV PA-injected animals had fewer cleaved caspase-3-positive cells in every 20 - $\mu \mathrm{m}$-thick section in the lesion (defined as the area marked by dense infiltration; $\boldsymbol{b}, \boldsymbol{d}$; ${ }^{* *} p<0.008$ by $t$ test) and as far as $400 \mu \mathrm{m}$ rostral and caudal to the lesion $\left(\boldsymbol{a}, \boldsymbol{d} ;{ }^{*} p<0.001\right.$ by $t$ test). c, Confocal Z-stacks of $20-\mu \mathrm{m}$-thick sections within $400 \mu \mathrm{m}$ of the lesion (red, $\mathrm{CC}_{\text {; }}$ blue, Hoechst) reveals an increased density of OLs in the IKVAV PA-injected animals. See text for quantitation. Scale bar, $20 \mu \mathrm{m}$.

\section{Effects of IKVAV PA on cell death}

The waves of apoptotic cell death that occur after SCI are also detrimental to functional recovery (Crowe et al., 1997; Beattie et al., 2002). We quantified cell death late in the second phase of apoptosis using cleaved caspase- 3 immunohistochemistry at $10 \mathrm{~d}$ after SCI (Liu et al., 1997; Beattie et al., 2002). In IKVAV PAinjected animals, there were significantly fewer cells undergoing apoptosis both in the area of maximal cell infiltration (lesion; $44.7 \pm 3.06$ for vehicle vs $34.3 \pm 2.02$ for IKVAV PA; $p<0.008$ ) (Fig. $5 b, d$ ) and on either side of the lesion (30.8 \pm 4.26 for vehicle and $16.3 \pm 1.25$ for IKVAV PA; $p<0.001$ ) (Fig. 5a,d). Because there is typically significant oligodendroglial (OL) death at this stage after injury (Beattie et al., 2002), we used CC1 immunohistochemistry (Beattie et al., 2002) to measure OL density in the same area adjacent to the lesion, where we found a decreased incidence of cell death after IKVAV PA injection. There was a significant increase in the number of CC1 + cells in the IKVAV PA-injected animals $\left(64.4 \pm 4.75\right.$ cells per $0.25 \mathrm{~mm}^{2}$ for vehicle vs $85.8 \pm 6.56$ after IKVAV PA; $p<0.025)$, suggesting enhanced OL survival (Fig. $5 c$ ). Thus, IKVAV PA injection increased OL cell numbers concurrent with reduced apoptotic cell death and reduction of glial scar formation.

\section{Tract tracing of motor axons}

We used tract-tracing techniques with BDA to determine whether the amphiphile injection could support regeneration of injured motor and sensory axons. Representative traces and pictures of corticospinal tract tracing from an IKVAV PA-injected and a vehicle-injected animal at 11 weeks after injury (Fig. $6 a-f$ ) illustrate the marked difference (Fig. $6 h$ ) between the treated and control groups. Almost $80 \%$ of all labeled corticospinal axons in the IKVAV PA group entered the lesion compared with 50\% of the fibers in control animals (Fig. 6h). No fibers in the control animals were ever detected as far as $25 \%$ of the way across the lesion. In contrast, $\sim 50 \%$ of the fibers in the treated group penetrated half of the way through the lesion, and $\sim 45 \%$ of the fibers penetrated three-quarters of the distance. Strikingly, $35 \%$ of all of the labeled corticospinal fibers actually grew through the lesion and entered the spinal cord caudal to the lesion. The fibers took meandering, unusual courses through the lesion site and terminated after growing through the lesion. Nevertheless, to further exclude the possibility of axon sparing, BDA labeling was examined at 2 weeks after injury in a separate group (Fig. $6 g$ ). At this time, axons were heavily labeled with BDA, indicating intact integrity of axoplasmic transport, and the number of labeled axons near the rostral end of lesion did not differ between IKVAV PAinjected and control animals or between the 2 week and 11 week groups. At 2 weeks after injury, no fibers in either group were ever observed even $25 \%$ of the way through the lesion, demonstrating that spared fibers were not present in any animals. Together, these findings strongly support the thesis that the bioactive nanofibers promoted regeneration of CST motor fibers.

\section{Tract tracing of sensory axons}

Representative traces and pictures of sensory axon tracing from IKVAV PA-injected and vehicle-injected animals at 11 weeks after injury (Fig. 7a-f) illustrate the marked difference between the treated and control groups that received BDA injections. Approximately $60 \%$ of labeled axons in the IKVAV PA group entered the lesion compared with only $\sim 20 \%$ of the fibers in controls (Fig. 7h). Only rare fibers in controls grew $25 \%$ of the way across the lesion, and no fibers in controls penetrated as far as $50 \%$. In contrast, $\sim 35 \%$ of the fibers in the treated group penetrated $25 \%$ of the way through the lesion, and $\sim 25 \%$ of the fibers penetrated $50 \%$ of the distance. Importantly, $\sim 10 \%$ of the fibers actually grew through the lesion and entered the spinal cord rostral to the lesion. Again to further exclude the possibility of axon sparing, BDA labeling was examined at 2 weeks after injury in a separate group (Fig. $7 g$ ). At this time, axons were heavily labeled with BDA, indicating the integrity of axoplasmic transport, and the number of labeled axons near the caudal end of lesion did not differ between IKVAV and control. At 2 weeks after injury, no fibers in either group were ever observed penetrating even as far as $25 \%$ of the way through the lesion, demonstrating that spared fibers were not present. In toto, these findings support the thesis 


\section{Descending Corticospinal Motor Axons}

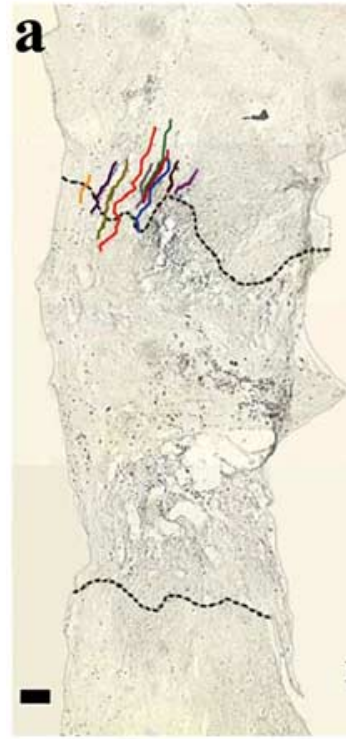

Control

g

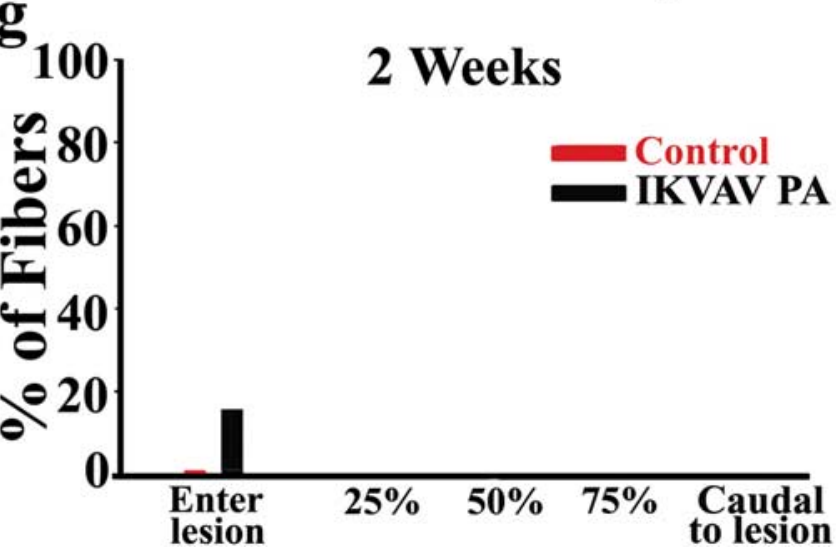

b
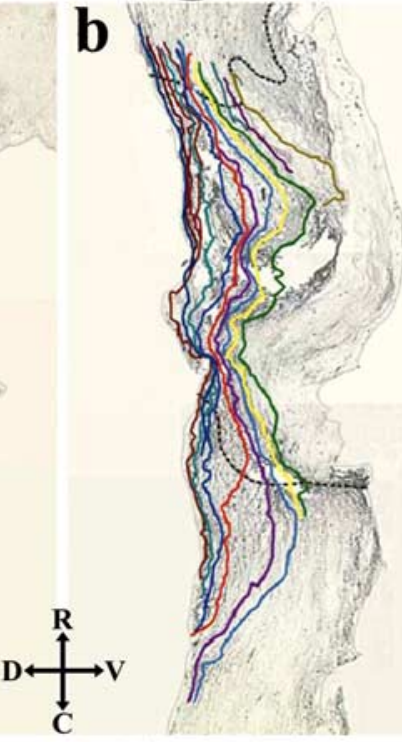

(1) C
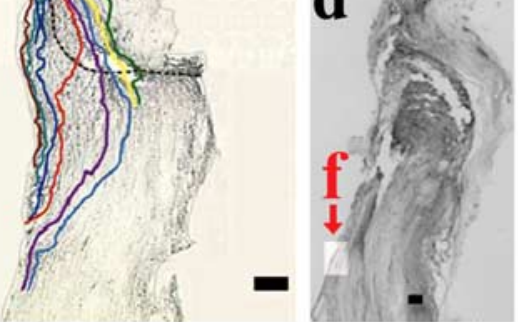

h

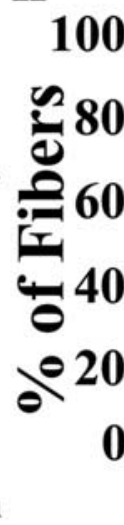

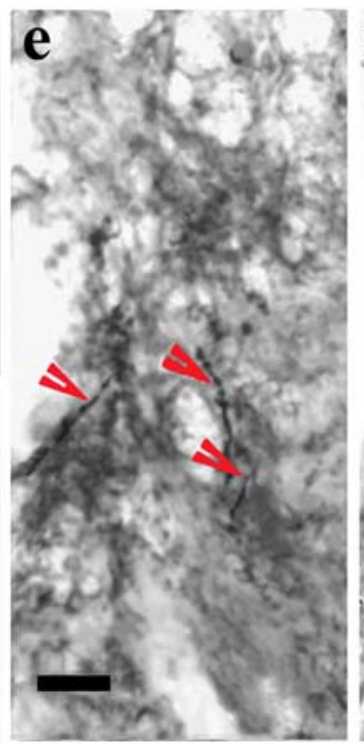

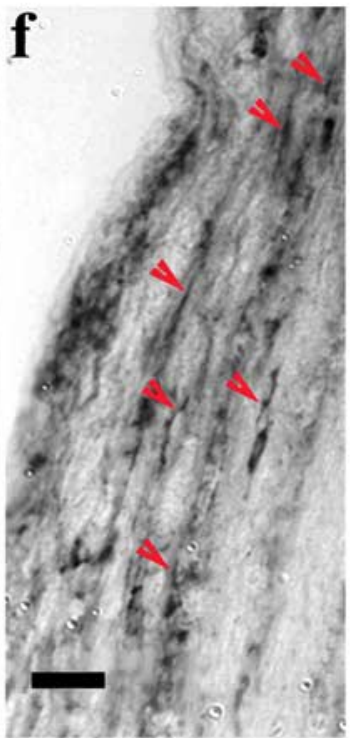

In Lesion Caudal to Lesion

11 Weeks

Figure 6. IKVAV PA promotes regeneration of motor axons after SCl. $\boldsymbol{a}, \boldsymbol{b}$, Representative Neurolucida tracings of BDA-labeled descending motor fibers within a distance of $500 \mu \mathrm{m}$ rostral of the lesion in vehicle-injected $(\boldsymbol{a})$ and IKVAV PA-injected $(\boldsymbol{b})$ animals. The dotted lines demarcate the borders of the lesion. $\boldsymbol{c}-\boldsymbol{f}$, Bright-field images of BDA-labeled tracts in lesion $(\boldsymbol{c}, \boldsymbol{e})$ and caudal to lesion $(\boldsymbol{d}, \boldsymbol{f})$ used for Neurolucida tracings in an IKVAV PA-injected spinal cord $(\boldsymbol{a}, \boldsymbol{b}) . \boldsymbol{g}, \boldsymbol{h}$, Bar graphs show the extent to which labeled corticospinal axons penetrated the lesion. ${ }^{*}$ The groups representing three control and three IKVAV PA mice and the tracing of 130 individual axons differ from each other at $p<0.03$ by the Wilcoxon rank test. R, Rostral; $\mathbf{C}$, caudal; D, dorsal; $\mathbf{V}$, ventral. Scale bars: $\boldsymbol{a}-\boldsymbol{d}, 100 \mu \mathrm{m} ; \boldsymbol{e}-\mathbf{f}, 25 \mu \mathrm{m}$.

that the bioactive nanofibers promoted regeneration of sensory fibers.

Illustration of the unusual course and morphology of traced axons

The Neurolucida drawings in Figures 6 and 7 are twodimensional reconstructions of a three-dimensional phenomenon and therefore do not completely demonstrate the unusual courses of the axons. To further illustrate this process, montages of sections and focal planes were assembled for a single representative axon from Figure 7 (Figs. 8, 9; supplemental Fig. 7, available at www.jneurosci.org as supplemental material). These fibers met the rigorous published criteria (Steward et al., 2003) for distinguishing regeneration from sparing of axons. Notably, the fibers took meandering, unusual courses through the injured tissue (Figs. 8, 9; supplemental Fig. 7, available at www.jneurosci.org as supplemental material), terminated at varying distances after passing through the lesion site, and displayed unusual morphologies not characteristic of normal axons (Figs. 7-9).

\section{Discussion}

Although adult CNS neurons have the intrinsic capacity to regenerate axons after injury (Richardson et al., 1980), axon elongation and functional recovery after SCI is limited because of an unfavorable extracellular milieu (Silver and Miller, 2004; Thuret et al., 2006). In this study, we sought to develop a clinically relevant technique for providing an environment that might foster recovery from injury. We specifically used an injury model that mimics the type of injury seen in the clinic and we implemented therapy with a delay after experimental injury that is relevant to the time elapsed from clinical injury to hospital or spinal stabilization. The efficacy of the IKVAV PA despite the severity of the injury and delay in treatment highlights its unique ability to provide an environment conducive to recovery after SCI.

\section{Cellular actions of the IKVAV PA}

In previous studies, we investigated PA containing the bioactive sequence, IKVAV, and the nonbioactive sequence EQS for their effects on cultured neural progenitor cells and neurons (Silva et 


\section{Ascending Dorsal Sensory Axons}
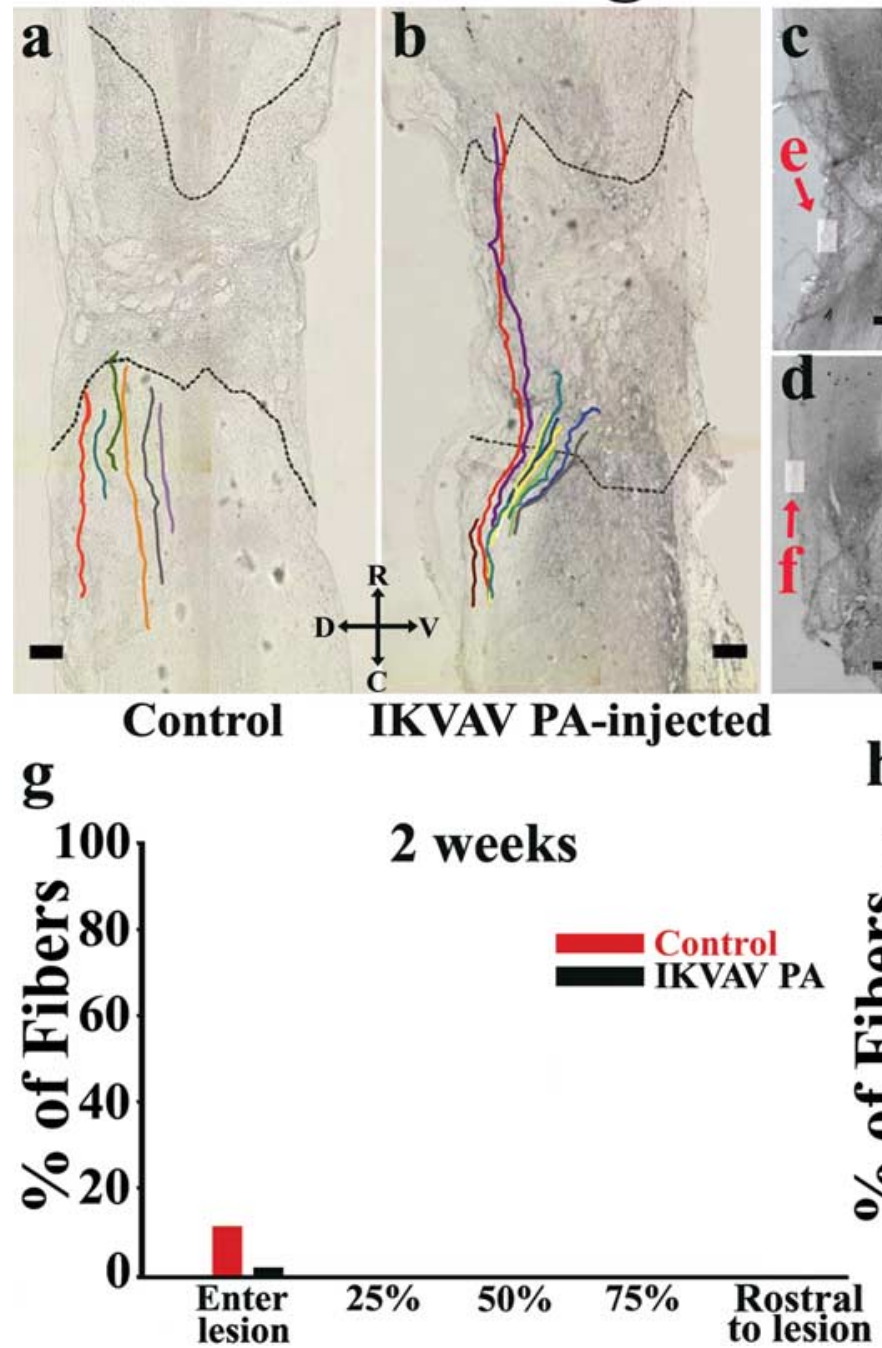

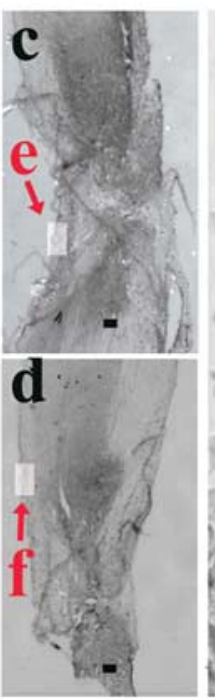

h

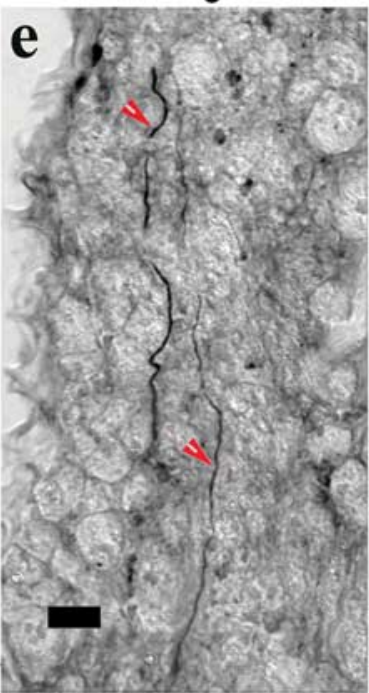

In Lesion

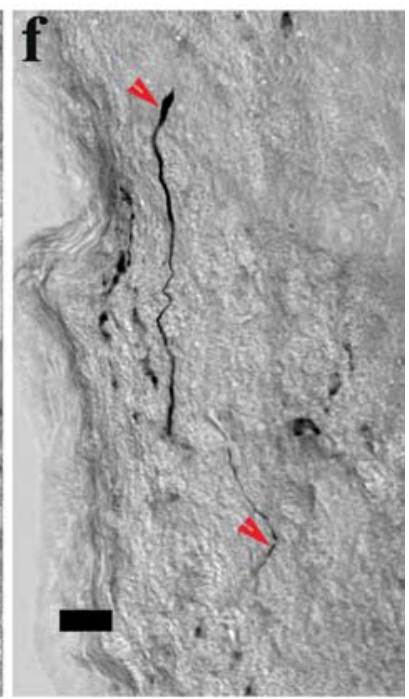

Rostral to Lesion

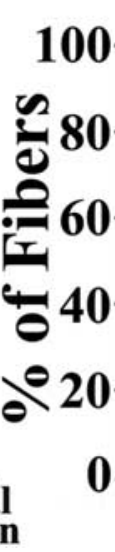

11 weeks

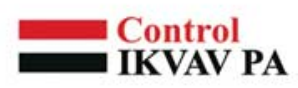

Figure 7. IKVAVPA promotes regeneration of sensory axons after SCl. $\boldsymbol{a}, \boldsymbol{b}$, Representative Neurolucida tracings of BDA-labeled ascending sensory fibers within a distance of $500 \mu \mathrm{m}$ of the lesion epicenter in vehicle-injected $(\boldsymbol{a})$ and IKVAV PA-injected $(\boldsymbol{b})$ animals. The dotted lines demarcate the borders of the lesion. $\boldsymbol{c}-\boldsymbol{f}$, Bright-field images of BDA-labeled tracts in lesion ( $\boldsymbol{c}, \boldsymbol{e})$ and rostral to lesion $(\boldsymbol{d}, \boldsymbol{f})$ used for Neurolucida tracings in an IKVAV PA-injected spinal cord $(\boldsymbol{a}, \boldsymbol{b})$. The top arrowhead points to an axon tip that morphologically resembles a growth cone. $\boldsymbol{g}, \boldsymbol{h}$, Bar graphs showing the extent to which labeled dorsal column axons entered and grew through the lesion. ${ }^{* *}$ The groups (representing 4 control and 4 IKVAV PA mice and the tracing of 185 individual axons) differ from each other at $p<0.05$ by the Wilcoxon rank test. R, Rostral; C, caudal; D, dorsal; V, ventral. Scale bars: $\boldsymbol{a}-\boldsymbol{d}, 100 \mu \mathrm{m} ; \boldsymbol{e}-\boldsymbol{f}, 25 \mu \mathrm{m}$.

al., 2004). As predicted, the IKVAV PA promoted copious outgrowth of neurites from cultured neurons (Silva et al., 2004). Unexpectedly, however, the IKVAV PA suppressed astroglial differentiation of cultured neural progenitor cells (Silva et al., 2004). The IKVAV sequence was necessary for the effects of the molecule on progenitor cells and neurons. However the IKVAV PA exerted effects strikingly different from either IKVAV peptide alone or laminin, indicating that the nanostructure of the molecule as well as the IKVAV sequence is necessary for its biological actions (Silva et al., 2004) (supplemental Fig. 5, available at www.jneurosci.org as supplemental material). This may reflect the extraordinary density of epitope presented by the matrix, estimated to be $10^{3}$-fold greater than that of laminin (Silva et al., 2004). Interestingly, the IKVAV PA did not alter the initial astrocytic hypertrophy after injury, indicating that this signaling mechanism does not affect hypertrophy and process extension in astrocytes. In turn, this suggests a duality of signaling pathways that regulate astrocytic hypertrophy versus hyperplasia. Although formation of the astroglial scar is a major impediment to axonal regeneration after SCI, astrogliosis after CNS injury also serves beneficial functions, such as repairing the blood-brain barrier and reducing infiltration by circulating cells (Bush et al., 1999; Faulkner et al., 2004; Okada et al., 2006), which may be critical for restoring homeostasis. These effects have been attributed to the initial reactive hypertrophy (Bush et al., 1999; Faulkner et al., 2004; Okada et al., 2006), which is unaltered in the IKVAV PA-injected animals. Thus the IKVAV PA appears to allow these beneficial events to occur while nevertheless limiting the progression of gliosis that impedes axon outgrowth.

The IKVAV nanofibers do not alter survival of cultured neural cells, and it is thus unclear why they ameliorated apoptosis in the injured spinal cord. Injection of the PA increased the number of OLs in the injured spinal cord, and it seems likely that this reflects enhanced survival of OLs that undergo apoptosis after SCI (Schwab and Bartholdi, 1996; Popovich et al., 1997; Dumont et al., 2001). However, it is also possible that the IKVAV PA fostered generation of OLs from precursor cells. Regardless of the mech- 


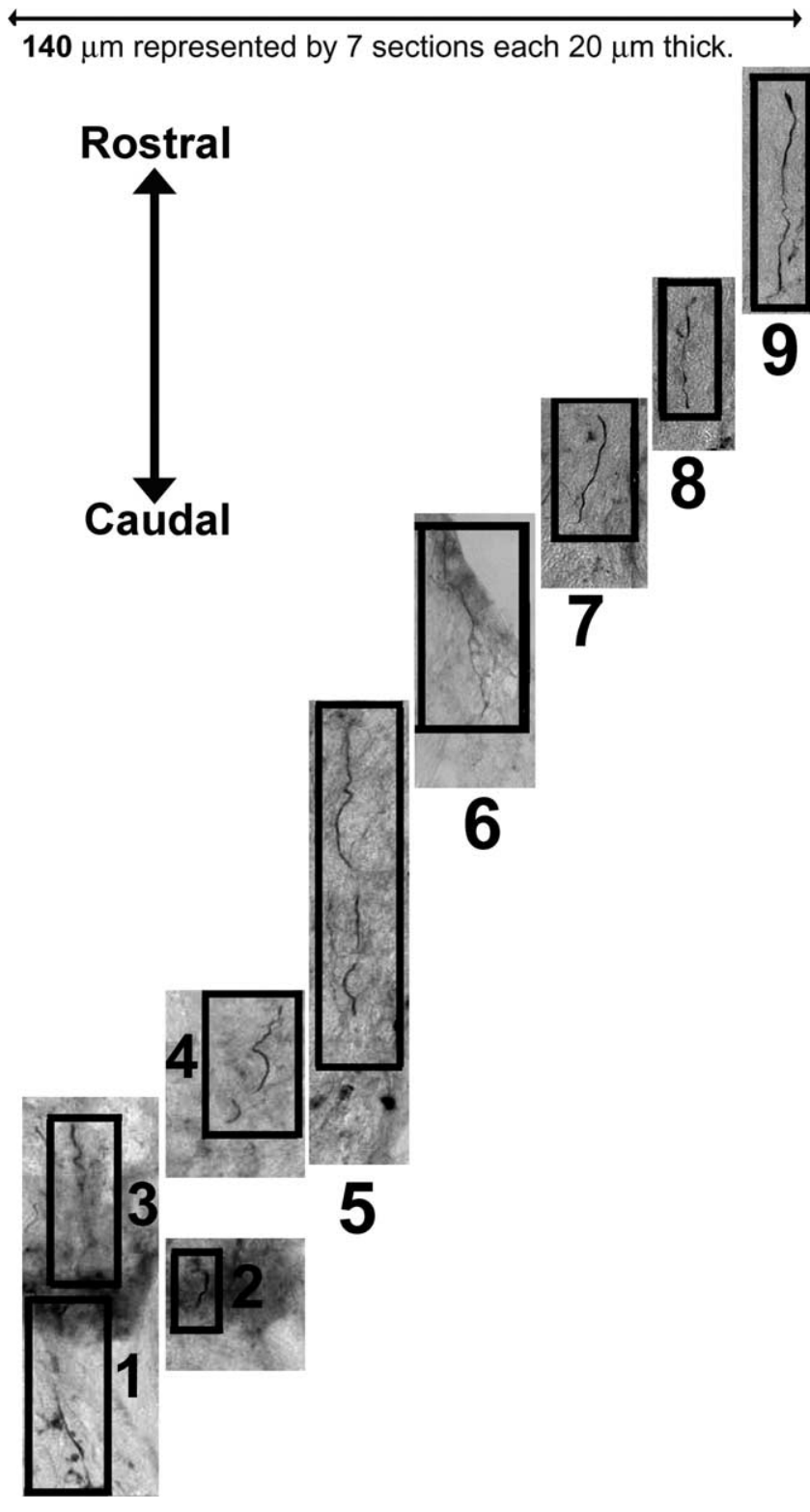

Figure 8. Montage of spinal cord sections illustrates the third dimension of axon regeneration. The Neurolucida drawings in Figures 6 and 7 are two-dimensional reconstructions of a three-dimensional phenomenon, and they therefore do not fully demonstrate the very unusual courses and morphologies of axons passing through the lesioned area. To further demonstrate the type of path taken by axons in the IKVAV PA-treated lesions, seven bright-field sections containing a representative axon from Figure 7 were assembled (for orientation, see supplemental Fig. 7, available at www.jneurosci.org as supplemental material). These seven sections represent only a small part of the total of 94 sections for this spinal cord. Note the meandering, unusual course of the fiber through the injured tissue and that it migrates out of and back into individual sections and across different sections. Also note that the fiber terminates rostral to the lesion after passing through the lesion site. The numbers label the individual images of the combined montage in Figure 9 and in supplemental Figure 7 (available at www.jneurosci.org as supplemental material).

anisms, the increased number of OLs at the site of injury could facilitate remyelination of axons that traverse the lesion (Keirstead and Blakemore, 1999; Keirstead et al., 2005; KarimiAbdolrezaee et al., 2006).

\section{Tract tracing}

Tract tracing demonstrated that both motor and sensory axons were able to traverse the area of injury in the treated spinal cord.

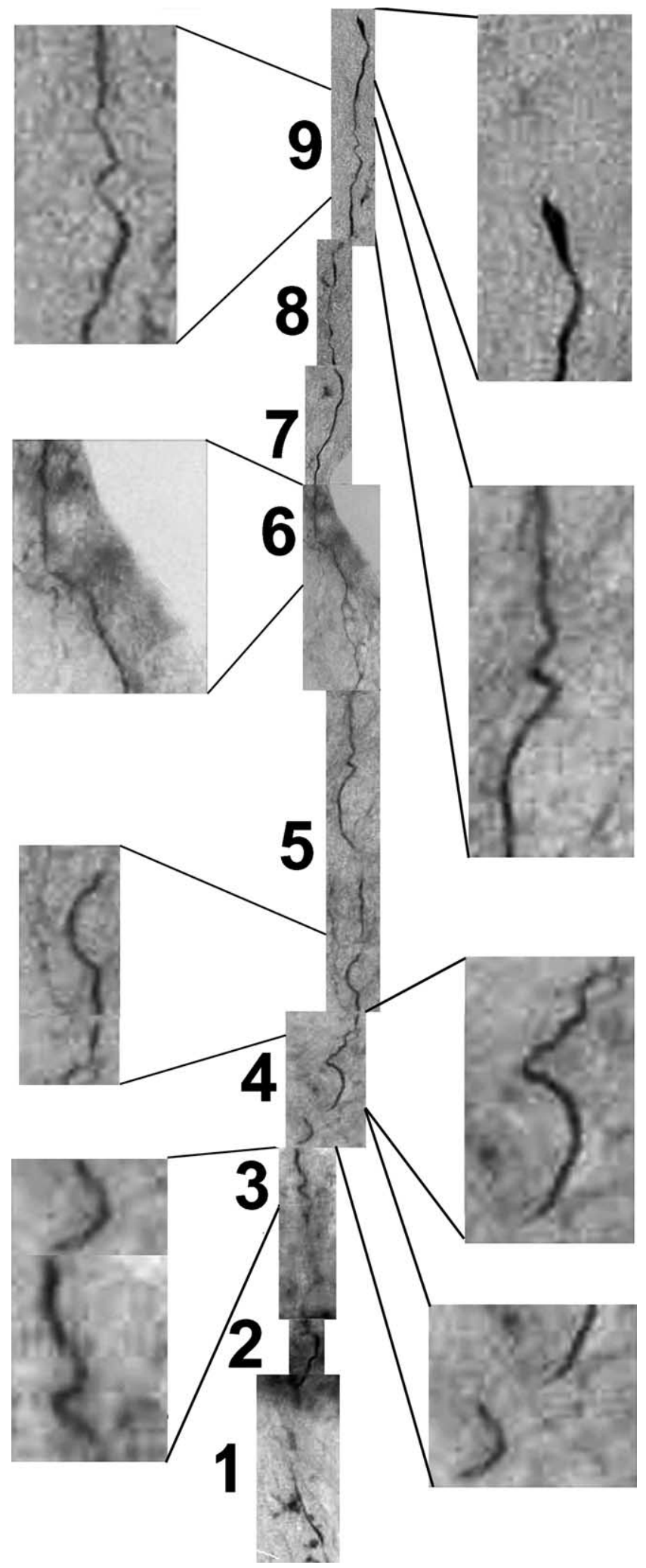

Figure 9. Combined montage flattens the representative axon to two dimensions to demonstrate the unusual morphology of the axon as it passes through the lesion. The Neurolucida tracings in Figures 6 and 7 follow the course of individual axons in three dimensions by tracing them from section to section and displaying their courses in a usable two-dimensional form. The drawing does not reflect the unusual morphologies of the axons. To demonstrate this, the separate sections of Figure 8 were aligned in a montage to display the course of a single axon as it starts at the caudal end of the lesion, passes through the lesion, and terminates rostral to the lesion. Note the unusual course and morphology, and that the axon terminates within normal tissue rostral to the lesion. 
These axons originated near the site of injury, took unusual courses through the injury site, extended at a plausible rate (3-11 weeks to traverse the injury site), and displayed unusual morphologies not characteristic of normal axons (Figs. 6-9). Importantly, all labeled axons terminated at variable distances after crossing the lesion, making the sparing of axons unlikely (Steward et al., 2003), particularly in view of the severity of injury. Thus, these axons fulfilled the published criteria for distinguishing spared from regenerated axons (Steward et al., 2003). Furthermore, no axons could be detected traversing the injury site 2 weeks after the injury, strongly mitigating against the possibility that there were spared axons, although this possibility cannot be totally excluded. The regeneration of these fibers occurred between 2 weeks and 11 weeks after injury; however, the stability of the nanofibers in the spinal cord is on the order of weeks. Therefore, this regeneration must have occurred during or after the degradation of the material. We propose two possible explanations for the axonal elongation. First, many of the effects of the $\mathrm{PA}$, including the increase in the number of oligodendrocytes, the reduction in cell death, and the initial suppression of astrogliosis, occur before degradation of the material. It is thus possible that some of the effects on axon elongation are secondary to these changes. Second, our previous publication demonstrated that the PA promotes an extraordinarily rapid effect on neurite production in vitro (Silva et al., 2004), and it is possible that in vivo the intracellular responses of neurons to the PA persist beyond the lifetime of detectable material in the spinal cord.

The timing of the degradation of the IKVAV PA may be important for its success in facilitating growth of fibers through the lesion site. Permanent grafts using Schwann cells or other nonbiodegradable materials can effectively promote axonal growth into, but not through, the transplanted material or tissue, because regenerating axons will not exit the favorable microenvironment of the graft (Bunge, 2001). In contrast, degradation of the IKVAV PA after ingrowth of axons may have allowed regenerating axons to continue to grow past the lesion site.

\section{Artificial scaffolds and SCI}

Numerous previous studies have used a variety of natural (e.g., collagen, agarose, and alginates) and synthetic (e.g., poly $\alpha$-hydroxy acids and polyvinylchloride) polymers for repair of the damaged spinal cord or brain (Teng et al., 2002; Stokols and Tuszynski, 2004, 2006; Jain et al., 2006). In some instances, the scaffolds were coated with laminin or other constituents of the ECM before implantation into the nervous system. A unique feature of the PA used in this study is its ability to self-assemble into nanofibers after being injected as a liquid into the spinal cord. This material can be injected into an uninjured spinal cord without behavioral sequellae (our unpublished observations). Another unique feature that distinguishes it from most previous scaffolds is that a signaling sequence has been incorporated into its backbone to enable it to act in an instructive manner on surrounding cells. This is an important advance, because it allows us to use biologically relevant epitopes, like the IKVAV sequence, that elicit responses different from those of the parent molecule. Notably the IKVAV PA caused cellular changes, including reductions in both gliosis and cell death, as far away as $400 \mu \mathrm{m}$ from the injection site.

Although the IKVAV PA promoted functional improvement, only partial recovery was seen, and the relationship between the behavioral improvement and the observed axon regeneration is unclear. Molecular structuring of the molecule or supramolecular structuring of the nanofibers to include other bioactive se- quences in addition to IKVAV (Hartgerink et al., 2002) or use in conjunction with other strategies may potentially further enhance its therapeutic efficacy (Thuret et al., 2006). Optimization of other characteristics of the material, such as its half-life in vivo and its mechanical characteristics, may also enhance its biological activity.

\section{References}

Basso DM, Beattie MS, Bresnahan JC, Anderson DK, Faden AI, Gruner JA, Holford TR, Hsu CY, Noble LJ, Nockels R, Perot PL, Salzman SK, Young W (1996) MASCIS evaluation of open field locomotor scores: effects of experience and teamwork on reliability. Multicenter animal spinal cord injury study. J Neurotrauma 13:343-359.

Beattie MS, Harrington AW, Lee R, Kim JY, Boyce SL, Longo FM, Bresnahan JC, Hempstead BL, Yoon SO (2002) ProNGF induces p75-mediated death of oligodendrocytes following spinal cord injury. Neuron 36:375-386.

Bunge MB (2001) Bridging areas of injury in the spinal cord. Neuroscientist 7:325-339.

Bush TG, Puvanachandra N, Horner CH, Polito A, Ostenfeld T, Svendsen CN, Mucke L, Johnson MH, Sofroniew MV (1999) Leukocyte infiltration, neuronal degeneration, and neurite outgrowth after ablation of scar-forming, reactive astrocytes in adult transgenic mice. Neuron 23:297-308.

Crowe MJ, Bresnahan JC, Shuman SL, Masters JN, Beattie MS (1997) Apoptosis and delayed degeneration after spinal cord injury in rats and monkeys. Nat Med 3:73-76.

Dumont RJ, Okonkwo DO, Verma S, Hurlbert RJ, Boulos PT, Ellegala DB, Dumont AS (2001) Acute spinal cord injury, part I: pathophysiologic mechanisms. Clin Neuropharmacol 24:254-264.

Faulkner JR, Herrmann JE, Woo MJ, Tansey KE, Doan NB, Sofroniew MV (2004) Reactive astrocytes protect tissue and preserve function after spinal cord injury. J Neurosci 24:2143-2155.

Fawcett JW, Asher RA (1999) The glial scar and central nervous system repair. Brain Res Bull 49:377-391.

Hartgerink JD, Beniash E, Stupp SI (2001) Self-assembly and mineralization of peptide-amphiphile nanofibers. Science 294:1684-1688.

Hartgerink JD, Beniash E, Stupp SI (2002) Peptide-amphiphile nanofibers: a versatile scaffold for the preparation of self-assembling materials. Proc Natl Acad Sci USA 99:5133-5138.

Jain A, Kim YT, McKeon RJ, Bellamkonda RV (2006) In situ gelling hydrogels for conformal repair of spinal cord defects, and local delivery of BDNF after spinal cord injury. Biomaterials 27:497-504.

Jiang H, Guler MO, Stupp SI (2007) The internal structure of self-assembled amphiphile nanofibers. Soft Matter 3:454-462.

Joshi M, Fehlings MG (2002a) Development and characterization of a novel, graded model of clip compressive spinal cord injury in the mouse: Part 1. Clip design, behavioral outcomes, and histopathology. J Neurotrauma 19:175-190.

Joshi M, Fehlings MG (2002b) Development and characterization of a novel, graded model of clip compressive spinal cord injury in the mouse: Part 2. Quantitative neuroanatomical assessment and analysis of the relationships between axonal tracts, residual tissue, and locomotor recovery. J Neurotrauma 19:191-203.

Karimi-Abdolrezaee S, Eftekharpour E, Wang J, Morshead CM, Fehlings MG (2006) Delayed transplantation of adult neural precursor cells promotes remyelination and functional neurological recovery after spinal cord injury. J Neurosci 26:3377-3389.

Keirstead HS, Blakemore WF (1999) The role of oligodendrocytes and oligodendrocyte progenitors in CNS remyelination. Adv Exp Med Biol 468:183-197.

Keirstead HS, Nistor G, Bernal G, Totoiu M, Cloutier F, Sharp K, Steward O (2005) Human embryonic stem cell-derived oligodendrocyte progenitor cell transplants remyelinate and restore locomotion after spinal cord injury. J Neurosci 25:4694-4705.

Liu XZ, Xu XM, Hu R, Du C, Zhang SX, McDonald JW, Dong HX, Wu YJ, Fan GS, Jacquin MF, Hsu CY, Choi DW (1997) Neuronal and glial apoptosis after traumatic spinal cord injury. J Neurosci 17:5395-5406.

Okada S, Nakamura M, Katoh H, Miyao T, Shimazaki T, Ishii K, Yamane J, Yoshimura A, Iwamoto Y, Toyama Y, Okano H (2006) Conditional ab- 
lation of Stat 3 or Socs 3 discloses a dual role for reactive astrocytes after spinal cord injury. Nat Med 12:829-834.

Popovich PG, Wei P, Stokes BT (1997) Cellular inflammatory response after spinal cord injury in Sprague-Dawley and Lewis rats. J Comp Neurol 377:443-464.

Richardson PM, McGuinness UM, Aguayo AJ (1980) Axons from CNS neurons regenerate into PNS grafts. Nature 284:264-265.

Schwab ME, Bartholdi D (1996) Degeneration and regeneration of axons in the lesioned spinal cord. Physiol Rev 76:319-370.

Silva GA, Czeisler C, Niece KL, Beniash E, Harrington DA, Kessler JA, Stupp SI (2004) Selective differentiation of neural progenitor cells by highepitope density nanofibers. Science 303:1352-1355.

Silver J, Miller JH (2004) Regeneration beyond the glial scar. Nat Rev Neurosci 5:146-156.

Steward O, Zheng B, Tessier-Lavigne M (2003) False resurrections: distin- guishing regenerated from spared axons in the injured central nervous system. J Comp Neurol 459:1-8.

Stokols S, Tuszynski MH (2004) The fabrication and characterization of linearly oriented nerve guidance scaffolds for spinal cord injury. Biomaterials 25:5839-5846.

Stokols S, Tuszynski MH (2006) Freeze-dried agarose scaffolds with uniaxial channels stimulate and guide linear axonal growth following spinal cord injury. Biomaterials 27:443-451.

Teng YD, Lavik EB, Qu X, Park KI, Ourednik J, Zurakowski D, Langer R, Snyder EY (2002) Functional recovery following traumatic spinal cord injury mediated by a unique polymer scaffold seeded with neural stem cells. Proc Natl Acad Sci USA 99:3024-3029.

Thuret S, Moon LD, Gage FH (2006) Therapeutic interventions after spinal cord injury. Nat Rev Neurosci 7:628-643. 